\title{
Colloidal charge reversal: Dependence on the ionic size and the electrolyte concentration
}

Cite as: J. Chem. Phys. 129, 124506 (2008); https://doi.org/10.1063/1.2982163

Submitted: 15 May 2008 . Accepted: 25 August 2008 . Published Online: 26 September 2008

Alexandre Diehl, and Yan Levin

\section{ARTICLES YOU MAY BE INTERESTED IN}

Smoluchowski equation and the colloidal charge reversal

The Journal of Chemical Physics 125, 054902 (2006); https://doi.org/10.1063/1.2222372

Overcharging and charge reversal in the electrical double layer around the point of zero charge

The Journal of Chemical Physics 132, 054903 (2010); https://doi.org/10.1063/1.3294555

Colloidal charge renormalization in suspensions containing multivalent electrolyte

The Journal of Chemical Physics 132, 104105 (2010); https://doi.org/10.1063/1.3354120

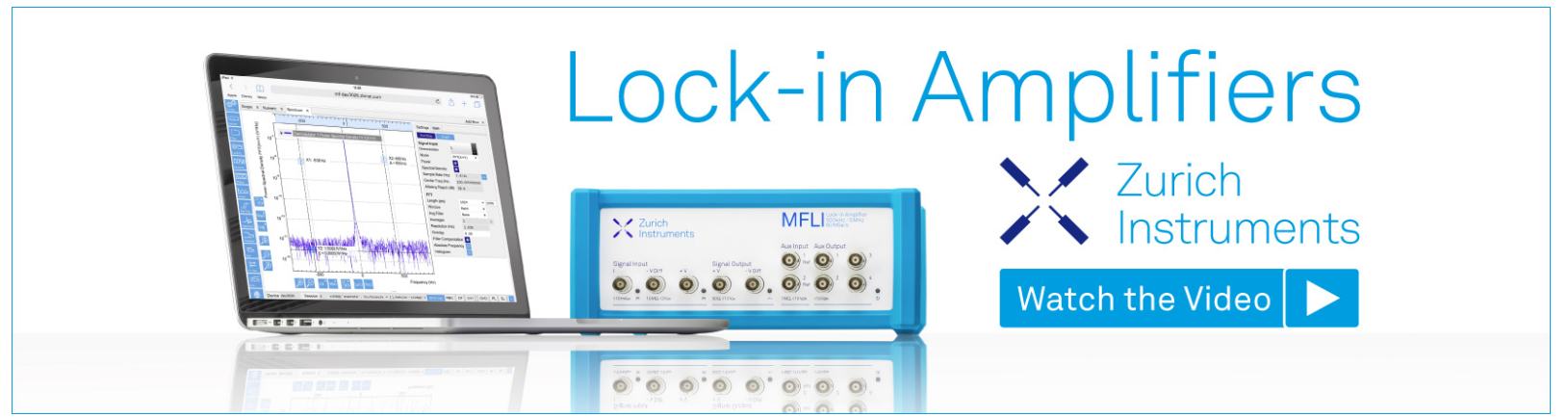

J. Chem. Phys. 129, 124506 (2008); https://doi.org/10.1063/1.2982163 


\title{
Colloidal charge reversal: Dependence on the ionic size and the electrolyte concentration
}

\author{
Alexandre Diehl ${ }^{1, a)}$ and Yan Levin ${ }^{2, b)}$ \\ ${ }_{1}^{1}$ Departamento de Física, Instituto de Física e Matemática, Universidade Federal de Pelotas, \\ Caixa Postal 354, Pelotas, Rio Grande do Sul 96010-900, Brazil \\ ${ }^{2}$ Instituto de Física, Universidade Federal do Rio Grande do Sul, Caixa Postal 15051, Porto Alegre, \\ Rio Grande do Sul 91501-970, Brazil
}

(Received 15 May 2008; accepted 25 August 2008; published online 26 September 2008)

\begin{abstract}
Extensive Monte Carlo simulations and scaling arguments are used to study the colloidal charge reversal. The critical colloidal surface charge density $\sigma_{c}$ at which the reversal first appears is found to depend strongly on the ionic size. We find that $\sigma_{c}$ has an inflection point as a function of the electrolyte concentration. The width of the plateau region in the vicinity of the inflection point depends on the temperature and the ionic radius $a$. In agreement with the theoretical predictions it is found that the critical colloidal charge above which the electrophoretic mobility becomes reversed diverges as $Z_{c} \sim 1 / a^{2}$ in the limit $a \rightarrow 0$. (C) 2008 American Institute of Physics.
\end{abstract}

[DOI: $10.1063 / 1.2982163$ ]

\section{INTRODUCTION}

A common way to stabilize colloidal suspensions against flocculation and precipitation is by synthesizing particles with acidic groups on their surface. When placed in water, these groups dissociate and colloids acquire a net negative charge. In aqueous suspension containing only monovalent electrolyte, the long range Coulomb repulsion prevents the colloids from approaching one another to distances for which the short range van der Waals interaction can lead to an irreversible sticking and precipitation. However, when beside the 1:1 electrolyte, suspensions contain some multivalent counterions, a number of curious and very counterintuitive effects can take place. ${ }^{1}$ For example, it has been observed that in such suspensions two like-charged colloidal particles can attract one another. ${ }^{2-16}$ This attraction is not a result of the van der Waals interaction, but rather a consequence of strong positional correlations between the multivalent counterions surrounding the colloidal particles. ${ }^{17-21}$ The mechanism of this attraction has been studied extensively, yet a fully predictive theory of this phenomenon still remains elusive.

Another curious effect observed in dilute colloidal suspensions containing multivalent counterions is the reversal of the electrophoretic mobility. ${ }^{1,22-24}$ Since the bare charge of colloidal particles is negative, when the electrostatic potential gradient is established in the suspension, one naturally expects that the particles should move in the direction opposite to the established electric field. Yet, what is often found is quite the opposite-particles drift in the direction of the field. ${ }^{25-27}$ The reversal of the electrophoretic mobility is a consequence of strong electrostatic interaction between the colloidal particles and the multivalent counterions. ${ }^{28-30}$ As a consequence of this coupling, some counterions become as-

\footnotetext{
${ }^{a)}$ Electronic mail: diehl@ufpel.edu.br.

${ }^{b)}$ Electronic mail: levin@if.ufrgs.br.
}

sociated (condensed) with the colloidal particle. The positional correlations induced by the electrostatic repulsion between the condensed counterions can lead to colloidcounterion complexes which are overcharged (charge reversed) - the number of condensed counterions can actually be larger than is necessary to completely neutralize the colloidal charge. ${ }^{1,31-35}$ If this happens, the electrophoretic mobility of colloidal particles will be reversed. While there are some theories which qualitatively account for this curious behavior, no fully predictive approach is yet available. ${ }^{34}$ In this paper we will use extensive Monte Carlo (MC) simulations to explore two aspects of this problem-the dependence of the minimum colloidal charge at which the reversal of the electrophoretic mobility takes place on: (one) the concentration of the multivalent $z: 1$ electrolyte and (two) on the ionic size.

\section{THE MODEL AND SIMULATIONS}

The electrophoretic mobility, in general, is a complicated nonlinear function of the electrokinetic $\zeta$ potential. ${ }^{36,37}$ For small $\zeta$ and large ionic strengths, however, the relationship between the two is linear and is given by the Smoluchowski equation. ${ }^{36,38}$ A change in the sign of the $\zeta$ potential will, therefore, lead to the reversal of the electrophoretic mobility, which we will also associate with the overcharging (or the charge reversal) of the colloidal particles. In principle, the overcharging (charge reversal) and the reversal of the electrophoretic mobility are two distinct concepts-one is static and the other dynamical. In practice, however, the definition of charge reversal carries some ambiguity. The general trends, such as the behavior of the effective charge and of the electrophoretic mobility as a function of, say, the ionic size or electrolyte concentration are very similar in two cases. ${ }^{39}$ We will, therefore, use the two concepts interchangibly. For a fixed electrolyte concentration, the value of the colloidal charge at which the $\zeta$-potential vanishes will be designated 
as the critical colloidal charge. Our goal is to find the dependence of this charge on the ionic size and the electrolyte concentration.

We consider a diluted aqueous mixture of colloidal particles inside a $z: 1$ electrolyte. The spherical colloidal particles have radius $R$ and surface charge $-Z q$, where $q$ is the elementary charge. For each colloidal particle there are $Z$ monovalent counterions. All the ions are modeled as hard spheres of radius $a$ with the charge $+z q$ (electrolyte counterion), $-q$ (electrolyte coion), or $+q$ (colloidal counterion) at their centers. The solvent is treated as a continuum of dielectric constant $\varepsilon$. The relative strength of the electrostatic interactions, as compared to the thermal energy, is measured by the ratio of the ionic radius to the Bjerrum length, $\lambda_{B}$ $=q^{2} / 4 \pi \varepsilon k_{B} T$.

As was argued in Ref. 39, the value of the $\zeta$ potential may be associated with the electrostatic potential at the effective shear plane removed from the colloidal surface by one ionic diameter. ${ }^{40}$ Since the maximum of the electrostatic potential also occurs at approximately the same position, the precise location of the shear plane does not influence strongly the value of the $\zeta$-potential. In this respect, our approach is quite similar to the one adopted by Bjerrum for simple electrolytes. ${ }^{41,42}$ The static potential at the effective shear plane can then be calculated using the canonical MC simulations. ${ }^{39}$ Recent simulations show the basic correctness of this picture for normal wetting surfaces. ${ }^{37,39}$ Working with $\zeta$-potential is also advantageous as compared to defining the effective charge in terms of condensed counterions located within a sheath surrounding the colloidal surface. Such definition carries a large degree of arbitrariness, since the condensed counterions will in turn drive a coassociations of coions. The effective charge will then be strongly sensitive to the precise value of the sheath width. This is not the case for $\zeta$-potential, which under the same conditions develops a maximum near the colloidal surface which diminishes its sensitivity to the precise location of the shear plane. Furthermore, since the $\zeta$-potential is calculated by integrating the electric field over the whole space, it already takes into account the layering effect that hinders the geometrical definition of the effective charge.

A colloidal particle is fixed at the center of a cubic simulation box of side length $L$ and is surrounded by the counterions and coions, the number of which satisfies the overall charge neutrality. We define $C$ as the molar concentration of the $z$-valent counterions derived from the dissociation of $z: 1$ (strong) electrolyte-assumed to be fully dissociated in an aqueous environment. The electrostatic interactions are computed using the Ewald summation method ${ }^{43}$ with 518 Fourier-space wave vectors and a real-space damping parameter $\kappa=5 / L$.

Two types of MC moves were utilized-ion transfer to a completely new random position inside the simulation box, which is useful for low salt concentrations, and a small linear displacement for high salt concentration, in order to give the standard acceptance ratios for the Metropolis algorithm. The number of microions in each simulation was varied from approximately 50 up to 3000 particles, depending on the molar salt concentration and the box length. Typical runs in- volved $10^{7} \mathrm{MC}$ steps for equilibration and $10^{8}$ steps for production. After equilibration, the average number of counterions and coions in concentric spherical shells of equal thickness around the colloid were accumulated in order to obtain the density profiles $\rho_{i}(r)$. The mean electrostatic potential at distance $r$ from the colloidal particle is then calculated as

$$
\phi(r)=\int_{r}^{\infty} d r^{\prime} E\left(r^{\prime}\right)=\frac{q}{4 \pi \varepsilon} \int_{r}^{\infty} d r^{\prime} \frac{P\left(r^{\prime}\right)}{r^{\prime 2}},
$$

where $E(r)$ is the electric field and $P(r)$ is the integrated charge (in units of $q$ ) within a distance $r$ from the center of the colloidal particle,

$$
P(r)=-Z+\int_{R}^{r}\left[\sum_{i} z_{i} \rho_{i}\left(r^{\prime}\right)\right] 4 \pi r^{\prime 2} d r^{\prime},
$$

where $i$ refers to the type of the microion. Since the typical integrated charge rapidly decays to zero, ${ }^{39}$ the upper cutoff in Eq. (1) is taken to be $L / 2$. Following Ref. 39 , the shear plane was located at one ionic diameter from the colloidal surface, so that $\zeta \equiv \phi\left(R_{s}\right)$, where $R_{s}=R+2 a$.

\section{SCALING ANALYSIS}

The model presented in the previous section is quite complex, with a number of distinct length scales. To organize and interpret the data of the MC simulations we shall, therefore, appeal to the dimensional and scaling analysis. There are five basic length scales: $R, L, a, \lambda_{B}$, and $C^{-1 / 3}$. Since we are interested in very dilute suspensions, $L \rightarrow \infty$. Although this limit cannot be achieved in the simulations, our box size was always taken to be sufficiently large so that critical colloidal charge $-Z_{c} q$ did not have any explicit dependence on $L$. We are, therefore, left with four relevant length scales, so that for a fixed $z: 1$ electrolyte, $Z_{c}$ is a function of only three dimensionless ratios,

$$
Z_{c}=f\left(\frac{a}{R}, \frac{a}{\lambda_{B}}, \lambda_{B} C^{1 / 3}\right),
$$

where $f(x, y, z)$ is a scaling function. Furthermore, we note that when the Debye length, $\xi_{D}=1 / \sqrt{4 \pi \lambda_{B}\left(z^{2}+z\right) C}$, is sufficiently short, $R / \xi_{D} \gg 1$-which is almost always the case near the isoelectric point-the curvature effects will be screened, and the critical colloidal charge must be proportional to the colloidal surface area. This means that

$$
Z_{c}=\frac{4 \pi R^{2}}{a^{2}} g\left(\frac{a}{\lambda_{B}}, \lambda_{B} C^{1 / 3}\right) .
$$

We conclude that for sufficiently large salt concentrations, the reversal of the electrophoretic mobility will take place when the modulus of the colloidal surface charge density, $\sigma \equiv Z q / 4 \pi R^{2}$, is larger than the critical value $\sigma_{c}$, which depends on the Bjerrum length, ionic radius, and the concentration of electrolyte through the scaling function $g(x, y)$, 


$$
\sigma_{c}=\frac{q}{a^{2}} g\left(\frac{a}{\lambda_{B}}, \lambda_{B} C^{1 / 3}\right) .
$$

The similarity transformation, Eq. (5), is particularly useful when one wants to obtain the critical surface charge density for suspensions with large concentrations of electrolyte. In these cases, the direct MC simulations become extremely slow due to large number of microions which must be used to simulate a dilute colloidal suspension in the $L \rightarrow \infty$ limit. However, Eq. (5) tells us that this critical surface charge density can also be obtained by simulating a much smaller system at a slightly lower temperature and with a somewhat larger microions. For example, suppose that we want to find the critical surface charge density of colloidal particles inside a dilute suspension at room temperature, $\lambda_{B}=7.2 \AA$, containing 3:1 electrolyte at concentration $C=1 \mathrm{M}$, with ions of radius of $2 \AA$. Instead of doing the direct simulation of this system, we can simulate a "similar"system with say half the number of microions, $C=0.5 \mathrm{M}$, at a slightly lower temperature, $\lambda_{B}=7.2 \times 2^{1 / 3}=9.07 \AA$ and with ions of radius of 2 $\times 2^{1 / 3}=2.5 \AA$. From Eq. (5), the critical charge of the original system (at concentration $C=1 M$ ) will be $2^{2 / 3}$ times the critical charge of the similar system. The latter simulations, however, are much easier to perform since the number of microions involved is much smaller.

In Refs. 44 and 34 it was argued that the critical surface charge density is determined by the work that must be performed to transfer a multivalent counterion from the bulk electrolyte to the colloidal surface. In particular, it was found that $\sigma_{c} \sim(\Delta \mu)^{2}$, where $\Delta \mu$ is the change in the ionic solvation free energy between the bulk and the colloidal surface. ${ }^{44}$ In the limit of vanishing $a, \Delta \mu$ diverges as $1 / a$. This divergence is a consequence of Bjerrum pairing of oppositely charged ions in the bulk electrolyte. ${ }^{1,41,45}$ The critical surface charge density, therefore, diverges as $\sigma_{c} \sim 1 / a^{2}$. Thus, the scaling function with $x=0, g(0, y)$, should be constant for all values of $y$. For small, but finite values of $x$, we expect to see a deviation from this behavior in two limits: When the entropic effects begin to dominate the electrostatics $C^{1 / 3} \lambda_{B}$ $<0.5$ and when the hard-core repulsion begins to dominates everything, $C^{1 / 3} a>0.2$.

\section{RESULTS AND DISCUSSION}

In Fig. 1 we show the $\zeta$-potential as a function of the colloidal charge density for a suspension containing 3:1 electrolyte at a molar concentration of $C=0.1 M$. The radius of the colloidal particle was fixed at $R=30 \AA$, while its charge $Z$ was varied. Most of the simulations were performed with the boxes of side length $L=120 \AA$, which is large enough to produce very small colloidal volume fractions, thus, minimizing the influence of the periodicity on the ionic distribution. However, for low salt concentrations we increased the box length to $L=150$ and $210 \AA$, in order to increase the number of particles and to obtain a better statistics of ion distribution around the colloid.

For weakly charged colloidal particles the increase (in modulus) in the surface charge density was accompanied by a uniform decline of the $\zeta$ potential $(\zeta$ accompanied the col-

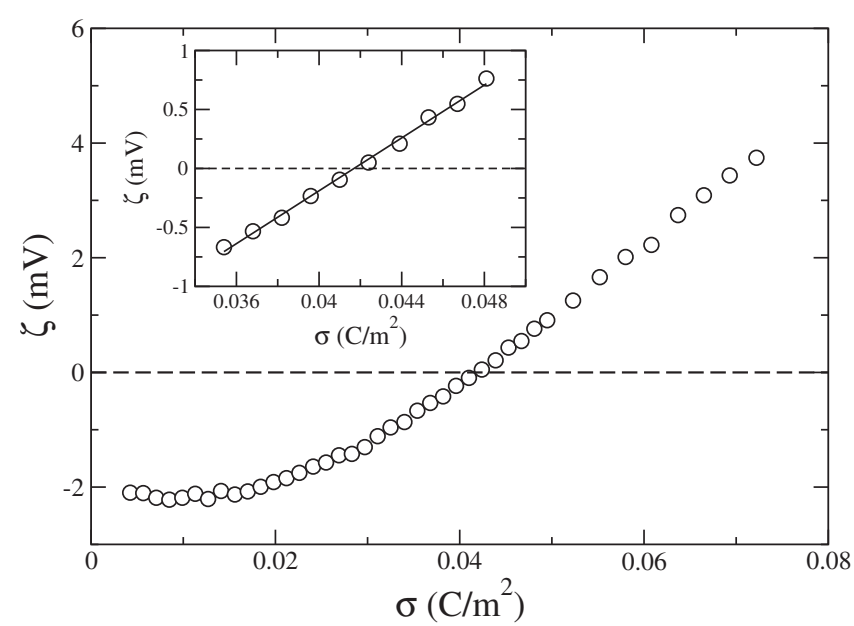

FIG. 1. Zeta potential as a function of the colloidal surface charge density. The molar concentration of $3: 1$ electrolyte is $C=0.1 M$, the radius of the microions is $a=3 \AA$, and the Bjerrum length is $7.2 \AA$. The inset shows the region where the $\zeta$ potential becomes reversed, $\zeta=0$, with a very good linear fit (solid line) to the simulation data (circles), from which the precise value of $\sigma_{c}$ is determined.

loidal charge and became more negative). However, when the colloidal charge became sufficiently large, counterion condensation became important and $\zeta$ increased as a function of the bare colloidal charge, becoming positive for sufficiently strongly charged colloids, see Fig. 1. To accurately determine the critical colloidal charge density $\sigma_{c}$ at which $\zeta=0$, we used a linear interpolation of the simulation data, as shown in the inset of Fig. 1.

We next studied the dependence of the critical surface charge density on the concentration of electrolyte. In Fig. 2, $\sigma_{c}$ is plotted as a function of $C$ for two different electrolytes: $a=2 \AA, \lambda_{B}=7.2 \AA$ and $a=3 \AA, \lambda_{B}=10.8 \AA$. Although clearly distinct, the two systems are similar, since the ratio $\lambda_{B} / a$ is the same in both cases. Therefore, if $\sigma_{c} a^{2} / q$ is plotted as a function of $\lambda_{B} C^{1 / 3}$ (or as a function of $C \lambda_{B}^{3}$ ) the data for the two systems should collapse onto a single curve. This is precisely what is found, see the inset of Fig. 2.

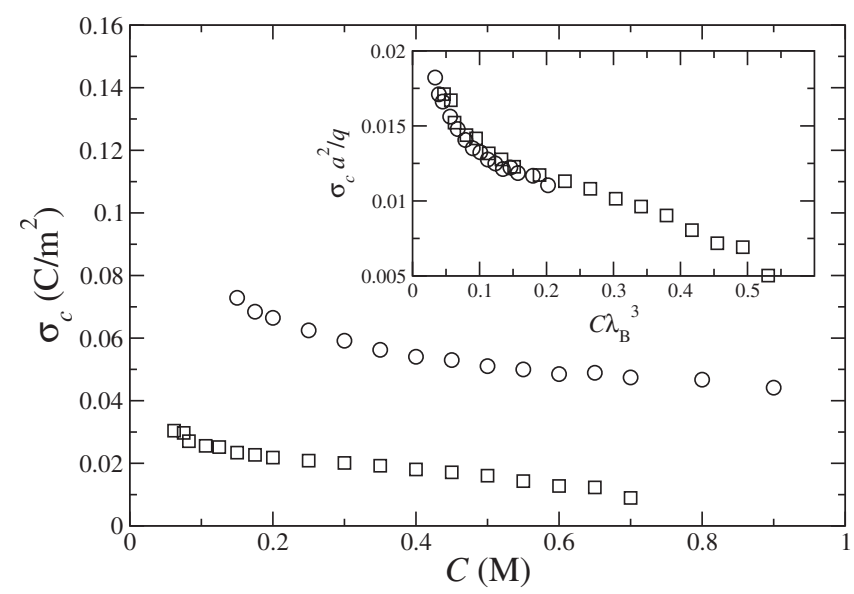

FIG. 2. Critical colloidal surface charge density as a function of the molar concentration of $3: 1$ electrolyte. The circles are for electrolyte with $a=2 \AA, \lambda_{B}=7.2 \AA$; the squares are for electrolyte with $a=3 \AA$, $\lambda_{B}=10.8 \AA$. In both cases the ratio $a / \lambda_{B}=0.278$ is the same. The inset shows the data collapse when the concentrations and $\sigma_{c}$ are properly scaled. 


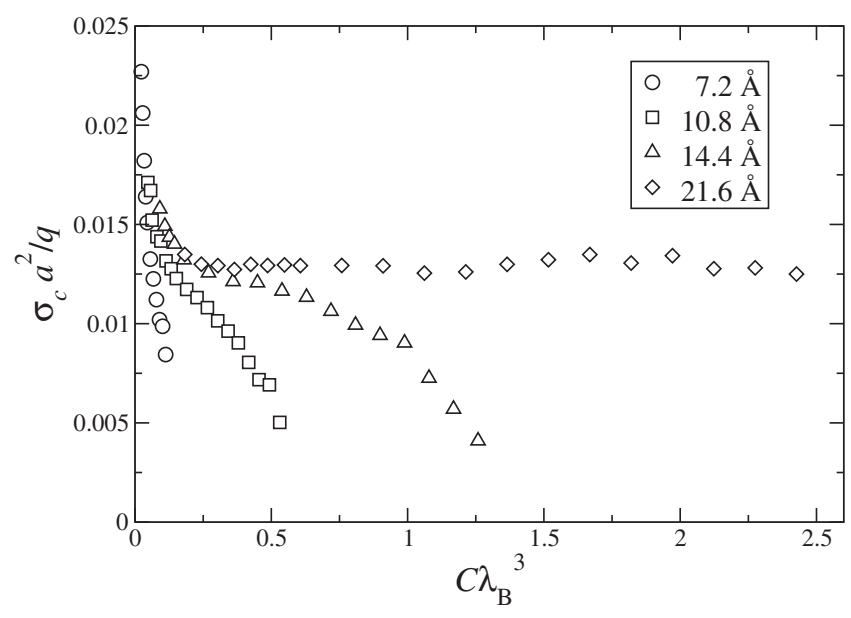

FIG. 3. Scaled critical colloidal surface charge density as a function of the scaled concentration of 3:1 electrolyte. The microions are of radius $a=3 \AA$.

If the electrolyte systems are not connected by the similarity transformation, a data collapse is not expected. Nevertheless, as was discussed in the previous section, if $a / \lambda_{B}$ is small, $g(x \approx 0, y)$ should be nearly constant. We expect, however, the deviation from this constancy to take place when the product $x y$ becomes sufficiently large - the separation between the microions becomes compatible to the ionic size, or when the concentration becomes so small that the entropic effects dominate over the electrostatics. In Fig. 3 we plot $\sigma_{c} a^{2} / q$ as a function $C \lambda_{B}^{3}$ for various electrolytes of different $\lambda_{B}$.

The figure clearly shows the inflection point of $\sigma_{c}$ as a function of the electrolyte concentration. Furthermore, in the limit of vanishing $a / \lambda_{B}$, the inflection point turns into plateau which extends up to the concentrations for which $C^{1 / 3} a \approx 0.2$, consistent with the discussion presented earlier. In the limit of vanishing ionic size, the plateau region extends indefinitely.

\section{CONCLUSIONS}

Using extensive MC simulations we have studied the dependence of the minimal colloidal charge at which the reversal of the electrophoretic mobility first takes place on the concentration of 3:1 electrolyte and on the ionic size. The critical surface charge density $\sigma_{c}$ was found to exhibit an inflection point as a function of the electrolyte concentration. In the limit of small $a / \lambda_{B}$, the inflection point becomes a flat plateau, extending from the lower concentration $C_{l}$ to the upper concentration $C_{u}$. The value of the lower bound is delimited by the distances at which the entropic effects begin to dominate over the electrostatics, $C_{l}^{1 / 3} \lambda_{B} \approx 0.5$, while the value of the upper bound is determined by the distances at which the hard-core repulsion begins to dominate over everything $C_{u}^{1 / 3} a \approx 0.2$. In the interval $\left[C_{l}, C_{u}\right]$ the surface charge density is found to be $\sigma_{c} \approx 0.013 q / a^{2}$, independent of the electrolyte concentration. The plateau disappears, turns into a simple inflection point with vanishing second derivative, when $\lambda_{B} / a<2.5$. The same behavior was found to occur for electrolytes of other valences. For example, in Fig. 4 we plot the critical surface charge density as a function the

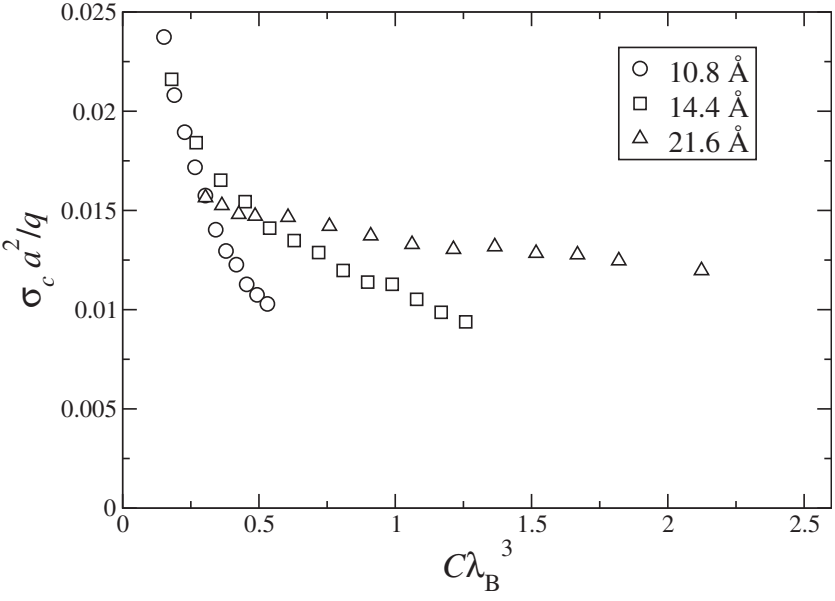

FIG. 4. Scaled critical colloidal surface charge density as a function of the scaled concentration of $2: 1$ electrolyte. The microions have radius $a=2 \AA$.

electrolyte concentration for 2:1 electrolyte. Once again the inflection point and the formation of the plateau are evident. However, in this case, to compensate for the weaker electrostatic interactions between the coions and the counterions, the value of $\lambda_{B}$ must be significantly lower for the plateau to appear clearly. What is surprising, however, is that the scaled surface charge density $\sigma_{c} a^{2} / q$ appears not to depend on the valence of the electrolyte-or depend only very weakly-in the plateau region. In the case of 2:1 electrolyte we find that $\sigma_{c} a^{2} / q \approx 0.015$ as compared to the 0.013 for the $3: 1$ electrolyte. It will be interesting to see if this curious behavior persists for other values of $z$. At the moment, there is no theory which can quantitatively account for these curious findings. We hope that the present study will provide a simulational benchmark against which the future theoretical predictions can be tested.

\section{ACKNOWLEDGMENTS}

This work is partially supported by $\mathrm{CNPq}$ and by the US-AFOSR under the Grant No. FA9550-06-1-0345.

${ }^{1}$ Y. Levin, Rep. Prog. Phys. 65, 1577 (2002).

${ }^{2}$ G. N. Patey, J. Chem. Phys. 72, 5763 (1980).

${ }^{3}$ R. Kjellander and S. Marcelja, J. Phys. Chem. 90, 1230 (1986)

${ }^{4}$ L. Guldbrand, B. Jonsson, H. Wennerstrom, and P. Linse, J. Chem. Phys. 80, 2221 (1984).

${ }^{5}$ J. M. Crocker and D. G. Grier, Phys. Rev. Lett. 73, 352 (1994).

${ }^{6}$ N. Grønbech-Jensen, R. J. Mashl, R. F. Bruinsma, and W. M. Gelbart, Phys. Rev. Lett. 78, 2477 (1997).

${ }^{7}$ E. Allahyarov, I. D’Amico, and H. Löwen, Phys. Rev. Lett. 81, 1334 (1998).

${ }^{8}$ A. W. C. Lau, P. Pincus, D. Levine, and H. A. Fertig, Phys. Rev. E 63, 051604 (2001).

${ }^{9}$ D. G. Grier, J. Phys.: Condens. Matter 12, A85 (2000).

${ }^{10}$ A. Diehl, H. A. Carmona, and Y. Levin, Phys. Rev. E 64, 011804 (2001).

${ }^{11}$ V. Lobaskin, A. Lyubartsev, and P. Linse, Phys. Rev. E 63, 020401 (2001).

${ }^{12}$ A. Naji and R. R. Netz, Eur. Phys. J. E 13, 43 (2004).

${ }^{13}$ A. A. Kornyshev and S. Leikin, Phys. Rev. Lett. 82, 4138 (1999).

${ }^{14}$ I. Rouzina and V. Bloomfield, J. Chem. Phys. 100, 9977 (1996).

${ }^{15}$ W. M. Gelbart, R. F. Bruinsma, P. A. Pincus, and V. A. Parsegian, Phys. Today 53(9), 38 (2000).

${ }^{16}$ A. A. Kornyshev, D. J. Lee, S. Leikin, and A. Wynveen, Rev. Mod. Phys. 79, 943 (2007).

${ }^{17}$ M. J. Stevens and M. O. Robbins, Europhys. Lett. 12, 81 (1990). 
${ }^{18}$ A. Diehl, M. N. Tamashiro, M. C. Barbosa, and Y. Levin, Physica A 274, 433 (1999).

${ }^{19}$ Y. Levin, Physica A 265, 432 (1999).

${ }^{20}$ J. J. Arenzon, J. F. Stilck, and Y. Levin, Eur. Phys. J. B 12, 79 (1999).

${ }^{21}$ F. J. Solis and M. O. de la Cruz, Phys. Rev. E 60, 4496 (1999).

${ }^{22}$ A. Y. Grosberg, T. T. Nguyen, and B. I. Shklovskii, Rev. Mod. Phys. 74, 329 (2002).

${ }^{23}$ M. Tanaka and A. Y. Grosberg, J. Chem. Phys. 115, 567 (2001).

${ }^{24}$ F. J. Solis, J. Chem. Phys. 117, 9009 (2002).

${ }^{25}$ M. Quesada-Pérez, J. Callejas-Fernández, and R. Hidalgo-Álvarez, Adv. Colloid Interface Sci. 95, 295 (2002).

${ }^{26}$ A. Martin-Molina, M. Quesada-Perez, F. Galisteo-Gonzalez, and R. Hidalgo-Alvarez, J. Phys.: Condens. Matter 15, S3475 (2003).

${ }^{27}$ A. Fernandez-Nieves, A. Fernandez-Barbero, F. J. de las Nieves, and B. Vincent, J. Chem. Phys. 123, 054905 (2005)

${ }^{28}$ G. M. Torrie and J. P. Valleau, J. Chem. Phys. 73, 5807 (1980).

${ }^{29}$ M. Lozada-Cassou, R. Saavedra-Barrera, and D. Henderson, J. Chem. Phys. 77, 5150 (1982).

${ }^{30}$ M. Deserno, F. Jimenez-Angeles, C. Holm, and M. Lozada-Cassou, J. Phys. Chem. 105, 10983 (2001).

${ }^{31}$ B. I. Shklovskii, Phys. Rev. E 60, 5802 (1999).
${ }^{32}$ F. J. Solis and M. O. de la Cruz, Eur. Phys. J. E 4, 143 (2001).

${ }^{33}$ R. Messina, C. Holm, and K. Kremer, Phys. Rev. E 64, 021405 (2001).

${ }^{34}$ S. Pianegonda, M. C. Barbosa, and Y. Levin, Europhys. Lett. 71, 831 (2005).

${ }^{35}$ O. Lenz and C. Holm, Eur. Phys. J. E 26, 191 (2008).

${ }^{36}$ W. B. Russel, D. A. Saville, and W. R. Schowalter, in Colloidal Dispersions, edited by G. K. Batchelor (Cambridge University Press, Cambridge, 1989).

${ }^{37}$ L. Joly, C. Ybert, E. Trizac, and L. Bocquet, Phys. Rev. Lett. 93, 257805 (2004).

${ }^{38}$ R. J. Hunter, Zeta Potential in Colloidal Science: Principles and Applications (Academic, London, 1981).

${ }^{39}$ A. Diehl and Y. Levin, J. Chem. Phys. 125, 054902 (2006).

${ }^{40}$ V. Lobaskin, B. Dünweg, M. Medebach, T. Palberg, and C. Holm, Phys. Rev. Lett. 98, 176105 (2007).

${ }^{41}$ N. Bjerrum, K. Dan. Vidensk. Selsk. Mat. Fys. Medd. 7, 1 (1926).

${ }^{42}$ M. E. Fisher and B. P. Lee, Phys. Rev. Lett. 77, 3561 (1996).

${ }^{43}$ M. P. Allen and D. J. Tildesley, Computer Simulations of Liquids (Oxford University Press, Oxford, 1987).

${ }^{44}$ Y. Levin, J. Phys.: Condens. Matter 16, S2149 (2004).

${ }^{45}$ Y. Levin and M. E. Fisher, Physica A 225, 164 (1996). 